\title{
ON THE DIOPHANTINE NATURE OF THE ELEMENTS OF CANTOR SETS ARISING IN THE DYNAMICS OF CONTRACTED ROTATIONS
}

\author{
YANN BUGEAUD, DONG HAN KIM, MICHEL LAURENT AND ARNALDO NOGUEIRA
}

\begin{abstract}
We prove that these Cantor sets are made up of transcendental numbers, apart from their endpoints 0 and 1 , under some arithmetical assumptions on the data. To that purpose, we establish a criterion of linear independence over the field of algebraic numbers for the three numbers 1, a characteristic Sturmian number, and an arbitrary Sturmian number with the same slope.
\end{abstract}

\section{INTRODUCTION AND RESULTS}

A notorious open question in Diophantine approximation, formulated by Mahler [13], is to decide whether the middle-third Cantor set

$$
\mathcal{C}=\left\{\sum_{k \geq 1} \frac{x_{k}}{3^{k}} ; x_{k} \in\{0,2\}, \forall k \geq 1\right\},
$$

defined as the set of real numbers in $[0,1]$ having no digit 1 in their ternary expansion, contains irrational algebraic elements. Since one commonly believes that irrational algebraic numbers are normal to base 3 (as to every other integer base), it is expected that the answer to Mahler's question is negative, but we are very far away from being able to prove this. Recall that $\mathcal{C}$ can as well be dynamically defined: it is the attractor of the iterated function system (IFS) $\left\{\phi_{0}, \phi_{1}\right\}$, where

$$
\phi_{0}(x)=\frac{1}{3} x, \quad \phi_{1}(x)=\frac{1}{3} x+\frac{2}{3} .
$$

The main goal of the present paper is to exhibit a family of Cantor sets in $[0,1]$ which also naturally arise in dynamics, precisely in the dynamics of contracted rotations, and for which we can prove that they contain no algebraic elements, except 0 and 1.

Throughout this paper, $\lfloor x\rfloor$ denotes the integer part, $\lceil x\rceil$ the upper integer part, and $\{x\}$ the fractional part of a real number $x$.

Definition 1. Let $\lambda$ and $\delta$ be real numbers with $0<\lambda<1$ and $1-\lambda<\delta<1$. We call the map $f_{\lambda, \delta}$ defined by

a contracted rotation of $[0,1)$.

$$
f=f_{\lambda, \delta}: x \in[0,1) \mapsto\{\lambda x+\delta\}
$$

In particular, the map $f_{\lambda, \delta}$ is a 2-interval piecewise affine contraction on the interval $I=[0,1)$. Figure 1 shows the graph of $f_{\lambda, \delta}$.

The following facts concerning the dynamics of contracted rotations have been established in $[2,3,8,9,10,11]$. Each map $f=f_{\lambda, \delta}$ has a rotation number $\theta=\theta_{\lambda, \delta}$ satisfying $0<\theta<1$. For any given $\lambda$ in $(0,1)$, this rotation number is rational for almost all values of $\delta$ in $(1-\lambda, 1)$. In particular, it has been proved in [10] that $\theta$ is rational when $\lambda$ and

DK was supported by the National Research Foundation of Korea (NRF-2018R1A2B6001624).

2010 Mathematics Subject Classification: 11J91, 37E05. 


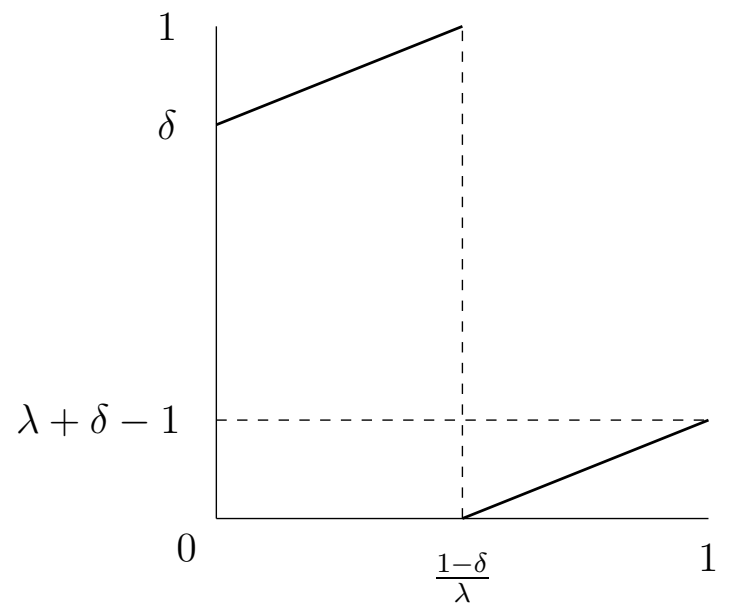

Figure 1. A plot of $f_{\lambda, \delta}: I \rightarrow I$

$\delta$ are both algebraic. On the other hand, if $\theta$ takes an irrational value, then the closure of the limit set $\bigcap_{n \geq 1} f^{n}(I)$, where $f^{n}$ stands for the $n$-th iterate of $f$, is a Cantor set $C=C_{\lambda, \delta}$. Then, any orbit $\left(f^{n}(x)\right)_{n \geq 0}$ asymptotically approaches of an orbit contained in $C$, as $n$ tends to infinity.

Moreover, for any real number $\lambda$ and any irrational number $\theta$ with $0<\lambda, \theta<1$, there exists one and only one value $\delta$ with $1-\lambda<\delta<1$ for which $\theta_{\lambda, \delta}=\theta$. This number $\delta$ is given by the series

$$
\delta=\delta(\lambda, \theta)=(1-\lambda)\left(1+\sum_{k \geq 1}(\lfloor(k+1) \theta\rfloor-\lfloor k \theta\rfloor) \lambda^{k}\right) .
$$

We are concerned with the Diophantine nature of the elements of the Cantor set $C_{\lambda, \delta(\lambda, \theta)}$. It is easily observed that the endpoints of $C_{\lambda, \delta(\lambda, \theta)}$ are 0 and 1 . Whenever $\lambda$ is an arbitrary algebraic number and $\theta$ an arbitrary irrational number, we expect that 0 and 1 are the only algebraic numbers contained in $C_{\lambda, \delta(\lambda, \theta)}$. Our first theorem confirms this expectation when $\lambda$ is the reciprocal of an integer.

Theorem 1. Let $b$ be an integer with $b \geq 2$ and $\theta$ an irrational number with $0<\theta<1$. Put $\lambda=1 / b$ and $\delta=\delta(\lambda, \theta)$. Then, any element of the Cantor set $C_{\lambda, \delta}$ which differs from its endpoints 0 and 1 is a transcendental number.

A key tool for the proof of Theorem 1 is a result of independent interest on linear independence of Sturmian numbers of the same slope.

Sturmian words are, by definition, the infinite aperiodic words of minimal complexity. They can be described as follows. Let $\theta$ and $\rho$ be real numbers with $0 \leq \theta, \rho<1$ and $\theta$ irrational. For $n \geq 0$, set

$$
s_{n}:=\lfloor(n+1) \theta+\rho\rfloor-\lfloor n \theta+\rho\rfloor, \quad s_{n}^{\prime}:=\lceil(n+1) \theta+\rho\rceil-\lceil n \theta+\rho\rceil .
$$

Then, the infinite words

$$
\boldsymbol{s}_{\theta, \rho}:=s_{0} s_{1} s_{2} \ldots, \quad \boldsymbol{s}_{\theta, \rho}^{\prime}:=s_{0}^{\prime} s_{1}^{\prime} s_{2}^{\prime} \ldots
$$

are, respectively, the lower and upper Sturmian words with slope $\theta$ and intercept $\rho$. Observe that $\boldsymbol{s}_{\theta, 0}$ and $\boldsymbol{s}_{\theta, 0}^{\prime}$ differ only by their first letter, thus, there exists an infinite word $\boldsymbol{c}_{\theta}$, called the characteristic Sturmian word with slope $\theta$, such that

$$
\boldsymbol{s}_{\theta, 0}=0 \boldsymbol{c}_{\theta}, \quad \boldsymbol{s}_{\theta, 0}^{\prime}=1 \boldsymbol{c}_{\theta} .
$$


Classical references on Sturmian words include [7, Chapter 6] and [12, Chapter 2]. If a and $\mathbf{b}$ are two distinct symbols, then a Sturmian sequence over $\{\mathbf{a}, \mathbf{b}\}$ is obtained from a Sturmian sequence over $\{0,1\}$ by replacing 0 by $\mathbf{a}$ and 1 by $\mathbf{b}$.

Let $b$ be an integer with $b \geq 2$. For an infinite word $\boldsymbol{x}=x_{1} x_{2} x_{3} \ldots$ over the alphabet $\{0,1, \ldots, b-1\}$, set

$$
\xi \boldsymbol{x}=\sum_{n=1}^{\infty} \frac{x_{n}}{b^{n}} .
$$

If $\boldsymbol{x}$ is a Sturmian word (of slope $\theta$ and intercept $\rho$ ) over two elements of the alphabet $\{0,1, \ldots, b-1\}$, then we call $\xi \boldsymbol{x}$ a Sturmian number (of slope $\theta$ and intercept $\rho$ ).

Ferenczi and Mauduit [6] established that every Sturmian number is transcendental. Their proof combines combinatorial properties of Sturmian words with a result from Diophantine approximation. Further progress has been made in the understanding of the combinatorial properties of Sturmian words, firstly by Berthé, Holton, and Zamboni [1], and subsequently by Bugeaud and Kim [4].

A careful analysis of the auxiliary lemmas obtained in [4] allows us to go one step further and to decide whether two Sturmian numbers of the same slope are linearly independent.

Theorem 2. Let $b$ be an integer with $b \geq 2$. Let $\mathbf{a}, \mathbf{b}$ be distinct integers in $\{0,1, \ldots, b-1\}$. Let $\theta$ and $\rho$ be real numbers with $0 \leq \theta, \rho<1$ and $\theta$ irrational. Let $\xi_{0}$ be a real number whose b-ary expansion is a characteristic Sturmian sequence of slope $\theta$ over $\{\mathbf{a}, \mathbf{b}\}$. Let $\xi_{1}$ be a real number whose b-ary expansion is a Sturmian sequence of slope $\theta$ and intercept $\rho$ over $\{\mathbf{a}, \mathbf{b}\}$. The real numbers $1, \xi_{0}, \xi_{1}$ are linearly independent over the field of algebraic numbers if and only if there does not exist an integer $j$ such that $\rho$ is congruent to $j \theta$ modulo one.

The 'only if' part is easy. Indeed, assume that $\theta$ in $(0,1)$ is an irrational number, $\rho$ is a real number and $j, k$ are integers such that $\rho=j \theta+k$. Then, for any positive integer $n$, we have

$$
\lfloor(n-j+1) \theta+\rho\rfloor-\lfloor(n-j) \theta+\rho\rfloor=\lfloor(n+1) \theta\rfloor-\lfloor n \theta\rfloor,
$$

and similarly if $\lfloor\cdot\rfloor$ is replaced by $\lceil\cdot\rceil$. This shows that, in this case, $\xi_{1}$ can be written as a linear combination of 1 and $\xi_{0}$ with rational coefficients.

It may well happen that 'linearly independent over the field of algebraic numbers' could be replaced by 'algebraically independent' in the theorem above, but such a result seems to be by far out of reach.

\section{Combinatorial properties of Sturmian words}

Let $\theta=\left[0 ; a_{1}, a_{2}, \ldots\right]$ be an irrational real number in $(0,1)$ with partial quotients $a_{1}, a_{2}, \ldots$ and convergents $p_{k} / q_{k}=\left[0 ; a_{1}, \ldots, a_{k}\right]$. The symbols $\mathbf{a}, \mathbf{b}$ are viewed as distinct elements of $\{0,1, \ldots, b-1\}$.

Let $\left(M_{k}\right)_{k \geq 1}$ be the sequence of finite words associated with $\left(a_{i}\right)_{i \geq 1}$, that is, defined by

$$
M_{0}=\mathbf{a}, \quad M_{1}=\mathbf{a}^{a_{1}-1} \mathbf{b}, \quad M_{k}=\left(M_{k-1}\right)^{a_{k}} M_{k-2}, \quad \text { for } k \geq 2 .
$$

Note that $\mathbf{a b}$ is a suffix of $M_{k}$ for any odd integer $k \geq 3$, while ba is a suffix of $M_{k}$ for any even integer $k \geq 2$. For $k \geq 2$, we denote by $M_{k}^{--}$the word $M_{k}$ deprived of its two last letters and we set $M_{k}^{*}=M_{k}^{--}$ba or $M_{k}^{*}=M_{k}^{--}$ab, according whether $k$ is odd or even. Thus, $M_{k}^{*}$ is the word which differs from $M_{k}$ by its last two letters and only by these letters. For instance, $M_{2}=\left(\mathbf{a}^{a_{1}-1} \mathbf{b}\right)^{a_{2}} \mathbf{a}$ and $M_{2}^{*}=\left(\mathbf{a}^{a_{1}-1} \mathbf{b}\right)^{a_{2}-1} \mathbf{a}^{a_{1}} \mathbf{b}$.

The commutation relation

$$
M_{k-1} M_{k}^{--}=M_{k} M_{k-1}^{--},
$$


holds for any $k \geq 3$, from which follows the formula

$$
M_{k}=M_{k-1} M_{k}^{\prime}=M_{k}^{\prime} M_{k-1}^{*},
$$

where we have set

$$
M_{k}^{\prime}=\left(M_{k-1}\right)^{a_{k}-1} M_{k-2} .
$$

Furthermore, the word $\lim _{k \rightarrow \infty} M_{k}=c_{1} c_{2} \ldots$ is the characteristic Sturmian word of slope $\theta$ and we set

$$
\xi_{0}=\sum_{n=1}^{\infty} \frac{c_{n}}{b^{n}} .
$$

Throughout this paper, $|W|$ denotes the length (number of letters) of the finite word $W$. Note that $\left|M_{k}\right|=q_{k}$. We begin with an easy lemma.

Lemma 2.1. Let $k \geq 3$ be an integer and $V$ a finite word over $\{\mathbf{a}, \mathbf{b}\}$. Put

$$
\boldsymbol{x}=V M_{k} M_{k} \cdots, \quad \boldsymbol{y}=V M_{k}^{\prime}\left(M_{k}\right)^{\infty} .
$$

Then, there exists a real number $\eta_{k}$ with $\left|\eta_{k}\right| \leq 1$ and

$$
\xi \boldsymbol{x}-\xi \boldsymbol{y}=\frac{(-1)^{k}(\mathbf{b}-\mathbf{a})(b-1)}{b^{|V|+q_{k}}}+\frac{(-1)^{k}(\mathbf{b}-\mathbf{a})(b-1)+\eta_{k}}{b^{|V|+2 q_{k}}} .
$$

In particular, if $V=\left(M_{k}\right)^{a} M_{k-1}$ for some nonnegative integer a, then

$$
\boldsymbol{y}=\left(M_{k}\right)^{a} M_{k-1} M_{k}^{\prime}\left(M_{k}\right)^{\infty}=\left(M_{k}\right)^{\infty}
$$

and

$$
\xi_{\boldsymbol{x}}-\xi_{\boldsymbol{y}}=\frac{(-1)^{k}(\mathbf{b}-\mathbf{a})(b-1)}{b^{(a+1) q_{k}+q_{k-1}}}+\frac{(-1)^{k}(\mathbf{b}-\mathbf{a})(b-1)+\eta_{k}}{b^{(a+2) q_{k}+q_{k-1}}} .
$$

Proof. By (4), the words

$$
V M_{k}^{\prime} M_{k-1}^{*} M_{k}^{\prime} M_{k-1}^{*} \quad \text { and } \quad V M_{k}^{\prime} M_{k-1} M_{k}^{\prime} M_{k-1}
$$

are prefixes of $\boldsymbol{x}$ and $\boldsymbol{y}$, respectively. Since $\mathbf{a b}$ and $\mathbf{b a}$ (respectively, ba and $\mathbf{a b}$ ) are suffixes of $M_{k-1}$ and $M_{k-1}^{*}$ for even $k$ (respectively, for odd $k$ ), this completes the proof.

Using Lemma 7.2 of [4], we have the following proposition. This is the key tool for the proof of Theorem 3 below.

Proposition 1. Let $k$ be an integer with $k \geq 3$ and $\boldsymbol{x}$ a Sturmian word over $\{\mathbf{a}, \mathbf{b}\}$ of slope $\theta=\left[0 ; a_{1}, a_{2}, \ldots\right]$. Then, there exist a uniquely determined non-empty suffix $U_{k}$ of $M_{k} M_{k+1}=\left(M_{k}\right)^{a_{k+1}+1} M_{k-1}$ and an integer $d_{k+1}$ such that

$$
d_{k+1} \in\left\{a_{k+1}, a_{k+1}+1\right\}
$$

and

$$
\boldsymbol{x}=U_{k}\left(M_{k}\right)^{d_{k+1}} M_{k-1} M_{k} M_{k+1}^{--} \cdots=U_{k}\left(M_{k}\right)^{d_{k+1}} M_{k-1} M_{k} M_{k} \ldots
$$

In particular, we have $U_{k}=M_{k+1}$ when $\boldsymbol{x}=\boldsymbol{c}_{\theta}$ is the characteristic Sturmian word.

Proof. By [4, Lemma 7.2], there exists a unique word $W$ satisfying

(i) $\boldsymbol{x}=W M_{k+1} M_{k} M_{k+1}^{--} \ldots$, where $W$ is a non-empty suffix of $M_{k+1}$, or

(ii) $\boldsymbol{x}=W M_{k} M_{k+1} M_{k} M_{k+1}^{--} \ldots$, where $W$ is a non-empty suffix of $M_{k+1}$, or

(iii) $\boldsymbol{x}=W M_{k+1} M_{k} M_{k+1}^{--} \ldots$, where $W$ is a non-empty suffix of $M_{k}$. 
This corresponds exactly to $2 q_{k+1}+q_{k}$ cases. Moreover, it was also shown in [4, Lemma $7.2]$ that the prefixes of length $2 q_{k+1}+q_{k}-1$ corresponding to these $2 q_{k+1}+q_{k}$ cases are all distinct.

For (i) and (ii), we put $U_{k}=W$. Then $U_{k}$ is a suffix of $M_{k} M_{k+1}$ and

$$
\boldsymbol{x}=U_{k}\left(M_{k}\right)^{a_{k+1}} M_{k-1} M_{k} M_{k+1}^{--} \ldots \quad \text { for (i) }
$$

and

$$
\boldsymbol{x}=U_{k}\left(M_{k}\right)^{a_{k+1}+1} M_{k-1} M_{k} M_{k+1}^{--} \ldots \quad \text { for (ii). }
$$

Note that the assumption $k \geq 3$ implies that $M_{k}$ is a prefix of $M_{k+1}^{--}$.

For (iii), we write $\boldsymbol{x}=W M_{k+1} M_{k} M_{k+1}^{--} \ldots$ where $W$ is a non-empty suffix of $M_{k}$. Let $\boldsymbol{x}^{\prime}=M_{k+1} M_{k} M_{k+1}^{--} \ldots$ be such that $\boldsymbol{x}=W \boldsymbol{x}^{\prime}$. Since $M_{k+1} M_{k} M_{k+1}^{--}=M_{k+1} M_{k+1} M_{k}^{--}$by (3), the Sturmian word $\boldsymbol{x}^{\prime}$ satisfies (i) or (ii) with $W=M_{k+1}$. Therefore, we have

$$
\boldsymbol{x}^{\prime}=M_{k+1} M_{k+1} M_{k} M_{k+1}^{--} \ldots
$$

or

$$
\boldsymbol{x}^{\prime}=M_{k+1} M_{k} M_{k+1} M_{k} M_{k+1}^{--} \ldots,
$$

depending on the $\left(2 q_{k+1}+q_{k}-1\right)$-th letter of $\boldsymbol{x}^{\prime}$.

We choose $U_{k}=W M_{k+1}$. Then $U_{k}$ is a suffix of $M_{k} M_{k+1}$ and

$$
\boldsymbol{x}=W \boldsymbol{x}^{\prime}=W M_{k+1} M_{k+1} M_{k} M_{k+1}^{--} \cdots=U_{k}\left(M_{k}\right)^{a_{k+1}} M_{k-1} M_{k} M_{k+1}^{--} \ldots
$$

or

$$
\boldsymbol{x}=W \boldsymbol{x}^{\prime}=W M_{k+1} M_{k} M_{k+1} M_{k} M_{k+1}^{--} \cdots=U_{k}\left(M_{k}\right)^{a_{k+1}+1} M_{k-1} M_{k} M_{k+1}^{--} \cdots
$$

For the characteristic Sturmian word $\boldsymbol{c}_{\theta}$, the recurrence relations (2) show that

$$
U_{k}=M_{k+1}=M_{k}^{a_{k+1}} M_{k-1}
$$

and that

$$
d_{k+1}=\left\{\begin{array}{lll}
a_{k+1} & \text { if } & a_{k+2} \geq 2 \\
a_{k+1}+1 & \text { if } & a_{k+2}=1
\end{array}\right.
$$

This concludes the proof of the proposition.

Throughout the rest of this section, we let $\boldsymbol{x}$ be a Sturmian word and keep the notation of Proposition 1.

Lemma 2.2. Set $c=(\mathbf{b}-\mathbf{a})(b-1)$. For a given $k \geq 3$, put $\boldsymbol{y}=U_{k}\left(M_{k}\right)^{\infty}$ and $\boldsymbol{y}^{\prime}=U_{k} M_{k}^{\prime}\left(M_{k}\right)^{\infty}$. Then there exist $\delta_{k}$ and $\delta_{k}^{\prime}$ in $(-1,1)$ satisfying

$$
\xi \boldsymbol{x}-\xi \boldsymbol{y}=\frac{(-1)^{k} c+\delta_{k} b^{-q_{k}+2}}{b^{\left|U_{k}\right|+\left(d_{k+1}+1\right) q_{k}+q_{k-1}}}, \quad \xi_{\boldsymbol{x}}-\xi_{\boldsymbol{y}^{\prime}}=\frac{(-1)^{k} c+\delta_{k}^{\prime} b^{-q_{k}+2}}{b^{\left|U_{k}\right|+q_{k}}} .
$$

Proof. Setting $V=U_{k}\left(M_{k}\right)^{d_{k+1}} M_{k-1}$, it follows from Lemma 2.1 that there exist $\eta_{k}$ with $\left|\eta_{k}\right| \leq 1$ and $\delta_{k}$ in $(-1,1)$ such that

$$
\begin{aligned}
\xi \boldsymbol{x}-\xi \boldsymbol{y} & =\frac{(-1)^{k}(\mathbf{b}-\mathbf{a})(b-1)}{b^{\left|U_{k}\right|+\left(d_{k+1}+1\right) q_{k}+q_{k-1}}}+\frac{(-1)^{k}(\mathbf{b}-\mathbf{a})(b-1)+\eta_{k}}{b^{\left|U_{k}\right|+\left(d_{k+1}+2\right) q_{k}+q_{k-1}}} \\
& =\frac{(-1)^{k} c+\delta_{k} b^{-q_{k}+2}}{b^{\left|U_{k}\right|+\left(d_{k+1}+1\right) q_{k}+q_{k-1}}} .
\end{aligned}
$$

Put $V=U_{k}$. Then,

$$
\boldsymbol{x}=U_{k}\left(M_{k}\right)^{d_{k+1}} M_{k-1} M_{k} M_{k} \cdots=V M_{k} M_{k} \ldots
$$


since $d_{k+1} \geq 1$ and $M_{k-1} M_{k}=M_{k} M_{k-1}^{*}$ by (3). It follows from Lemma 2.1 that there exist $\eta_{k}^{\prime}$ with $\left|\eta_{k}^{\prime}\right| \leq 1$ and $\delta_{k}^{\prime}$ in $(-1,1)$ such that

$$
\begin{aligned}
\xi \boldsymbol{x}-\xi_{\boldsymbol{y}^{\prime}} & =\frac{(-1)^{k}(\mathbf{b}-\mathbf{a})(b-1)}{b^{\left|U_{k}\right|+q_{k}}}+\frac{(-1)^{k}(\mathbf{b}-\mathbf{a})(b-1)+\eta_{k}^{\prime}}{b^{\left|U_{k}\right|+2 q_{k}}} \\
& =\frac{(-1)^{k} c+\delta_{k}^{\prime} b^{-q_{k}+2}}{b^{\left|U_{k}\right|+q_{k}}} .
\end{aligned}
$$

We deduce from (4) that the infinite word $\boldsymbol{y}^{\prime}=U_{k} M_{k}^{\prime}\left(M_{k}\right)^{\infty}$ is purely periodic with period length $q_{k}$. Note that for some integers $m_{k}, m_{k}^{\prime}$, we have

$$
\xi \boldsymbol{y}=\frac{m_{k}}{b^{\left|U_{k}\right|}\left(b^{q_{k}}-1\right)} \text { and } \xi \boldsymbol{y}^{\prime}=\frac{m_{k}^{\prime}}{b^{q_{k}}-1} .
$$

Set $\varepsilon=1 / 10$ and define

$$
r_{k}:= \begin{cases}\left|U_{k}\right|, \quad \text { if } \quad q_{k+1}>(1+\varepsilon)\left(\left|U_{k}\right|+q_{k}\right), \\ 0, & \text { if } \quad q_{k+1} \leq(1+\varepsilon)\left(\left|U_{k}\right|+q_{k}\right) .\end{cases}
$$

and

$$
t_{k}:=\left\{\begin{array}{lll}
d_{k+1} q_{k}+q_{k-1}, & \text { if } & q_{k+1}>(1+\varepsilon)\left(\left|U_{k}\right|+q_{k}\right), \\
\left|U_{k}\right|, & \text { if } & q_{k+1} \leq(1+\varepsilon)\left(\left|U_{k}\right|+q_{k}\right) .
\end{array}\right.
$$

Then for $k \geq 3$, there exist an integer $m_{k}$ and a real number $\delta_{k}$ with $\left|\delta_{k}\right|<1$ such that

$$
\xi \boldsymbol{x}-\frac{m_{k}}{b^{r_{k}}\left(b^{q_{k}}-1\right)}=\frac{(-1)^{k} c+\delta_{k} b^{-q_{k}+2}}{b^{r_{k}+q_{k}+t_{k}}} .
$$

In particular, for the real number $\xi_{0}$ defined in (5) from the characteristic Sturmian $\boldsymbol{c}_{\theta}$, we have $U_{k}=M_{k+1}=\left(M_{k}\right)^{a_{k+1}} M_{k-1}$ and there exist an integer $m_{k}^{0}$ and a real number $\delta_{k}^{0}$ with $\left|\delta_{k}^{0}\right|<1$ such that

$$
\xi_{0}-\frac{m_{k}^{0}}{b^{q_{k}}-1}=\frac{(-1)^{k} c+\delta_{k}^{0} b^{-q_{k}+2}}{b^{q_{k}+q_{k+1}}} .
$$

Lemma 2.3. Suppose that there are infinitely many indices $k$ with $a_{k} \geq 2$. Then, there are infinitely many indices $k$ such that

$$
q_{k+1}>(1+\varepsilon)(2+\varepsilon) q_{k} .
$$

Proof. If there are infinitely many $k$ with $a_{k} \geq 3$, then the lemma holds true since $(1+$ $\varepsilon)(2+\varepsilon)<3$. Otherwise, there exists $k_{0}$ such that $a_{k} \leq 2$ for $k \geq k_{0}$. Then, $q_{k-1}>q_{k} / 3$ for $k>k_{0}$. Consequently, if $a_{k+1}=2$ for some $k>k_{0}+1$, then

$$
q_{k+1}=2 q_{k}+q_{k-1}>\frac{7}{3} q_{k}>(1+\varepsilon)(2+\varepsilon) q_{k} .
$$

This completes the proof of the lemma.

Lemma 2.4. If $a_{k}=1$ for all large integers $k$, then there are infinitely many indices $k$ such that $\left|U_{k}\right|>(1+\varepsilon) q_{k}$.

Proof. Our assumption implies that $q_{k+1}>(1+\varepsilon) q_{k}$ for every large integer $k$. Suppose that $\left|U_{k}\right| \leq(1+\varepsilon) q_{k}$ for some large integer $k$. Then, $U_{k}$ is a suffix of $M_{k+1}$.

On the other hand, $U_{k+1}$ is a non-empty suffix of $M_{k+1} M_{k+2}=M_{k+1}^{2} M_{k}$. Thus, $U_{k+1}$ is either of the form $V M_{k+1} M_{k}$ or $V M_{k}$ for some non-empty suffix $V$ of $M_{k+1}=M_{k} M_{k-1}$, 
or $U_{k+1}$ is equal to a non-empty suffix $W$ of $M_{k}$. For the first case, Proposition 1 at levels $k$ and $k+1$ reads

$$
U_{k}\left(M_{k}\right)^{d_{k+1}} M_{k-1} \cdots=\boldsymbol{x}=V M_{k+1} M_{k}\left(M_{k+1}\right)^{d_{k+2}} \cdots=V M_{k} M_{k-1} \cdots
$$

which implies that $V=U_{k}$. For the second case, we have

$$
U_{k}\left(M_{k}\right)^{d_{k+1}} M_{k-1} \cdots=\boldsymbol{x}=V M_{k}\left(M_{k+1}\right)^{d_{k+2}} \cdots=V M_{k} M_{k} M_{k-1} \cdots
$$

which implies again that $V=U_{k}$. For the third case, Proposition 1 at level $k+1$, gives us the expression

$$
\boldsymbol{x}=W M_{k+1} M_{k+1} M_{k} M_{k+1} M_{k+1} \cdots=W M_{k+1} M_{k} M_{k-1} M_{k} M_{k} \cdots,
$$

when $d_{k+2}=2$, or

$$
\begin{aligned}
\boldsymbol{x}=W M_{k+1} M_{k} M_{k+1} M_{k+2}^{--} \ldots & =W M_{k+1} M_{k} M_{k} M_{k-1} M_{k} M_{k-1} M_{k}^{--} \cdots \\
& =W M_{k+1} M_{k} M_{k} M_{k-1} M_{k} M_{k} M_{k-1}^{--} \cdots,
\end{aligned}
$$

when $d_{k+2}=1$. Both expressions imply that $U_{k}=W M_{k+1}$, which is impossible since $U_{k}$ is assumed to be a suffix of $M_{k+1}$.

Hence, we conclude that either $U_{k+1}=U_{k} M_{k}$ or $U_{k+1}=U_{k} M_{k+1} M_{k}$. If $U_{k+1}=$ $U_{k} M_{k+1} M_{k}$, then $\left|U_{k+1}\right|>(1+\varepsilon) q_{k+1}$ and the lemma is established. Suppose that $U_{k+\ell}=U_{k+\ell-1} M_{k+\ell-1}$ for $\ell \geq 1$. Since

$$
\lim _{\ell \rightarrow \infty} \frac{\left|U_{k+\ell}\right|}{q_{k+\ell}}=\lim _{\ell \rightarrow \infty} \frac{\left|U_{k}\right|+q_{k}+\cdots+q_{k+\ell-1}}{q_{k+\ell}}=\frac{1+\sqrt{5}}{2},
$$

we have $\left|U_{k+\ell}\right|>(1+\varepsilon) q_{k+\ell}$ for some integer $\ell$. This concludes the proof of the lemma.

Lemma 2.5. For any Sturmian number $\xi \boldsymbol{x}$, the set $\mathcal{K}$ of integers $k$ satisfying

$$
t_{k}>q_{k}+\varepsilon\left(r_{k}+q_{k}\right), \quad q_{k+1}>(1+\varepsilon)\left(r_{k}+q_{k}\right),
$$

is infinite. Moreover, for any $k$ in $\mathcal{K}$, there exist integers $m_{k}^{0}, m_{k}$ such that

$$
\left|\xi_{0}-\frac{m_{k}^{0}}{b^{q_{k}}-1}\right| \leq \frac{1}{b^{r_{k}+2 q_{k}+\varepsilon\left(r_{k}+q_{k}\right)-2}}, \quad\left|\xi_{\boldsymbol{x}}-\frac{m_{k}}{b^{r_{k}}\left(b^{q_{k}}-1\right)}\right| \leq \frac{1}{b^{r_{k}+2 q_{k}+\varepsilon\left(r_{k}+q_{k}\right)-2}} .
$$

Proof. Let $k$ be such that

$$
q_{k+1}>(1+\varepsilon)\left(\left|U_{k}\right|+q_{k}\right)
$$

Then, we have

Thus,

$$
r_{k}=\left|U_{k}\right|, \quad t_{k}=d_{k+1} q_{k}+q_{k-1} .
$$

$$
t_{k}=d_{k+1} q_{k}+q_{k-1} \geq q_{k+1}>(1+\varepsilon)\left(r_{k}+q_{k}\right)>q_{k}+\varepsilon\left(r_{k}+q_{k}\right)
$$

and

$$
q_{k+1}>(1+\varepsilon)\left(\left|U_{k}\right|+q_{k}\right)=(1+\varepsilon)\left(r_{k}+q_{k}\right) .
$$

Consequently, the lemma holds true when (8) holds for infinitely many $k$.

Assume now that there exists $k_{0}$ such that $q_{k+1} \leq(1+\varepsilon)\left(\left|U_{k}\right|+q_{k}\right)$ for all $k>k_{0}$. Then, for $k>k_{0}$, we have

$$
r_{k}=0, \quad t_{k}=\left|U_{k}\right| \text {. }
$$

If $a_{k}=1$ for all large $k$, then we have $q_{k+1}>(1+\varepsilon) q_{k}$ for all large $k$ and by Lemma 2.4, there are infinitely many $k$ 's such that $t_{k}=\left|U_{k}\right|>(1+\varepsilon) q_{k}$. The remaining case is when there are infinitely many $k$ with $a_{k} \geq 2$. It then follows from Lemma 2.3 that there are infinitely many $k$ such that

$$
q_{k+1}>(1+\varepsilon)(2+\varepsilon) q_{k}
$$


For such an integer $k$, we have

and

$$
t_{k}=\left|U_{k}\right| \geq \frac{q_{k+1}}{1+\varepsilon}-q_{k}>(1+\varepsilon) q_{k}=q_{k}+\varepsilon\left(r_{k}+q_{k}\right)
$$

\[ q_{k+1}>(1+\varepsilon)(2+\varepsilon) \]
This shows that the set $\mathcal{K}$ is infinite.

Moreover, for any $k$ in $\mathcal{K}$, it follows from (6) and (7) that there exist integers $m_{k}^{0}, m_{k}$ such that

$$
\begin{gathered}
\left|\xi_{0}-\frac{m_{k}^{0}}{b^{q_{k}}-1}\right| \leq \frac{|c|+1}{b^{q_{k}+q_{k+1}}} \leq \frac{1}{b^{r_{k}+2 q_{k}+\varepsilon\left(r_{k}+q_{k}\right)-2}}, \\
\left|\xi_{\boldsymbol{x}}-\frac{m_{k}}{b^{r_{k}}\left(b^{q_{k}}-1\right)}\right| \leq \frac{|c|+1}{b^{r_{k}+q_{k}+t_{k}}} \leq \frac{1}{b^{r_{k}+2 q_{k}+\varepsilon\left(r_{k}+q_{k}\right)-2}} .
\end{gathered}
$$

This completes the proof of the lemma.

\section{Proof of Theorem 2}

We start by establishing a weaker version of Theorem 2, namely we consider linear independence over the set of rational numbers. Keep the notations of Section 2.

Theorem 3. The real numbers $1, \xi_{0}, \xi_{1}=\xi_{\boldsymbol{x}}$ are linearly independent over the rationals if and only if there does not exist an integer $j$ such that the intercept of $\boldsymbol{x}$ is congruent to $j \theta$ modulo one.

Proof. Let $z_{0}, z_{1}$ be nonzero integers. For any $k$ in $\mathcal{K}$, it follows from Lemma 2.5 that

$$
\left|z_{0} \xi_{0}+z_{1} \xi_{1}-\frac{z_{0} m_{k}^{0} b^{r_{k}}+z_{1} m_{k}}{b^{r_{k}}\left(b^{q_{k}}-1\right)}\right|<\left(\left|z_{0}\right|+\left|z_{1}\right|\right) b^{2-11\left(r_{k}+q_{k}\right) / 10}
$$

If $z_{0} \xi_{0}+z_{1} \xi_{1}$ is rational, then there exist infinitely many indices $k$ such that

$$
z_{0} \xi_{0}+z_{1} \xi_{1}=\frac{z_{0} m_{k}^{0} b^{r_{k}}+z_{1} m_{k}}{b^{r_{k}}\left(b^{q_{k}}-1\right)}
$$

that is, such that

$$
b^{r_{k}+t_{k}}\left((-1)^{k} c z_{0}+\delta_{k}^{0} z_{0} b^{-q_{k}+2}\right)+b^{q_{k+1}}\left((-1)^{k} c z_{1}+\delta_{k} z_{1} b^{-q_{k}+2}\right)=0,
$$

by (6) and (7). Since $b^{-q_{k}}$ tends to 0 as $k$ tends to infinity, we have

$$
b^{r_{k}+t_{k}} z_{0}+b^{q_{k+1}} z_{1}=0
$$

when $k$ is sufficiently large. Thus, there is an integer $C$ such that for arbitrarily large $k$

$$
r_{k}+t_{k}=q_{k+1}+C \text {. }
$$

Note that

$$
r_{k}+t_{k}= \begin{cases}\left|U_{k}\right|+d_{k+1} q_{k}+q_{k-1}, & \text { if } \quad q_{k+1}>(1+\varepsilon)\left(\left|U_{k}\right|+q_{k}\right), \\ \left|U_{k}\right|, & \text { if } \quad q_{k+1} \leq(1+\varepsilon)\left(\left|U_{k}\right|+q_{k}\right) .\end{cases}
$$

If $\left|U_{k}\right|+d_{k+1} q_{k}+q_{k-1}=q_{k+1}+C$ for infinitely many $k$, then $d_{k+1}=a_{k+1}$ and $\left|U_{k}\right|=C$ for infinitely many $k$. Otherwise, we deduce that

$$
\left|U_{k}\right|=q_{k+1}+C
$$

for infinitely many $k$. We thus distinguish three cases.

If $\left|U_{k}\right|=p$ for some integer $p>0$ and infinitely many $k$, then $U_{k}$ is the suffix of $M_{k-1}$ of length $p$. For arbitrary large $k$ we have $\boldsymbol{x}=U_{k} M_{k} \ldots$, where $\left|U_{k}\right|=p$ and $U_{k}$ is a suffix of $M_{k-1}$. Therefore, if we denote by $\sigma$ the left shift map, we find that $\sigma^{p}(\boldsymbol{x})=M_{k} \ldots$ for arbitrary large $k$. Thus, $\sigma^{p}(\boldsymbol{x})$ coincides with the characteristic Sturmian word $\boldsymbol{c}_{\theta}$. 
If $\left|U_{k}\right|=q_{k+1}+p$ for some integer $p \geq 0$ and infinitely many $k$, then $U_{k}=V M_{k+1}$ for some $V$ where $|V|=p$ and $V$ is a suffix of $M_{k}$. Since $\boldsymbol{x}=U_{k} \cdots=V M_{k+1} \ldots$ for arbitrary large $k$, it follows that $\sigma^{p}(\boldsymbol{x})=M_{k+1} \ldots$ for arbitrary large $k$, which is also the characteristic Sturmian word $\boldsymbol{c}_{\theta}$.

If $\left|U_{k}\right|=q_{k+1}-p$ for some integer $p \geq 0$ and infinitely many $k$, then $V U_{k}=M_{k+1}$ for some $V$ where $|V|=p$ and $V$ is a prefix of $M_{k+1}$. Since $V \boldsymbol{x}=V U_{k} \cdots=M_{k+1} \ldots$ for arbitrary large $k$, we now find the relation $\sigma^{p}\left(\boldsymbol{c}_{\theta}\right)=\boldsymbol{x}$.

It follows that the intercept of $\boldsymbol{x}$ is congruent to $( \pm p+1) \theta$ modulo one.

The other direction is clear.

We are in position to complete the proof of Theorem 2.

By [6], we already know that $\xi_{0}$ and $\xi_{1}$ are transcendental. Let $\alpha_{0}$ and $\alpha_{1}$ be non-zero real algebraic numbers. Our strategy is to apply the Subspace Theorem $[14,15]$ to prove that the real number $\alpha_{0} \xi_{0}+\alpha_{1} \xi_{1}$ is transcendental. We assume that it is algebraic and derive a contradiction.

Set $C=b^{2}\left(\left|\alpha_{0}\right|+\left|\alpha_{1}\right|\right)$. Let $k$ be in $\mathcal{K}$. Observe that, by Lemma 2.5, we have

$$
\left|\alpha_{0} \xi_{0}+\alpha_{1} \xi_{1}-\frac{\alpha_{0} m_{k}^{0}}{b^{q_{k}}-1}-\frac{\alpha_{1} m_{k}}{b^{r_{k}}\left(b^{q_{k}}-1\right)}\right|<\frac{C}{b^{r_{k}+2 q_{k}+\varepsilon\left(r_{k}+q_{k}\right)}} .
$$

Multiplying (9) by $b^{r_{k}}\left(b^{q_{k}}-1\right)$, we deduce that

$$
\left|\left(\alpha_{0} \xi_{0}+\alpha_{1} \xi_{1}\right) b^{r_{k}+q_{k}}-\left(\alpha_{0} \xi_{0}+\alpha_{1} \xi_{1}\right) b^{r_{k}}-\alpha_{0} m_{k}^{0} b^{r_{k}}-\alpha_{1} m_{k}\right|<\frac{C}{b^{q_{k}+\varepsilon\left(r_{k}+q_{k}\right)}} .
$$

Said differently, the linear form with algebraic coefficients

$$
L_{4}\left(X_{1}, X_{2}, X_{3}, X_{4}\right)=\left(\alpha_{0} \xi_{0}+\alpha_{1} \xi_{1}\right) X_{1}-\left(\alpha_{0} \xi_{0}+\alpha_{1} \xi_{1}\right) X_{2}-\alpha_{0} X_{3}-\alpha_{1} X_{4}
$$

takes small values at the integer quadruple $\left(b^{r_{k}+q_{k}}, b^{r_{k}}, m_{k}^{0} b^{r_{k}}, m_{k}\right)$. Define

$$
L_{1}\left(X_{1}, X_{2}, X_{3}, X_{4}\right)=X_{1}, \quad L_{2}\left(X_{1}, X_{2}, X_{3}, X_{4}\right)=X_{2}, \quad L_{3}\left(X_{1}, X_{2}, X_{3}, X_{4}\right)=X_{3} .
$$

Clearly, these four linear forms are linearly independent. For every prime divisor $\ell$ of $b$, set

$$
L_{i, \ell}\left(X_{1}, X_{2}, X_{3}, X_{4}\right)=X_{i}, \quad 1 \leq i \leq 4 .
$$

Using the obvious estimate $\left|m_{k}^{0}\right| \gg \ll b^{q_{k}}$ and (10), observe that there exist $\delta>0$ and infinitely many $k$ in $\mathcal{K}$ such that

$$
\prod_{j=1}^{4} \prod_{\ell \mid b}\left|L_{j, \ell}\left(b^{r_{k}+q_{k}}, b^{r_{k}}, m_{k}^{0} b^{r_{k}}, m_{k}\right)\right|_{\ell} \prod_{j=1}^{4}\left|L_{j}\left(b^{r_{k}+q_{k}}, b^{r_{k}}, m_{k}^{0} b^{r_{k}}, m_{k}\right)\right| \leq H_{k}^{-\delta},
$$

where $|.|_{\ell}$ stands for the usual $\ell$-adic absolute value on $\mathbb{Q}$, and where we have set

$$
H_{k}=\max \left\{b^{r_{k}+q_{k}}, b^{r_{k}},\left|m_{k}^{0}\right| b^{r_{k}},\left|m_{k}\right|\right\} .
$$

Then, the Subspace Theorem $[14,15]$ asserts that there exists a proper rational subspace of $\mathbb{Q}^{4}$ containing infinitely many quadruples $\left(b^{r_{k}+q_{k}}, b^{r_{k}}, m_{k}^{0} b^{r_{k}}, m_{k}\right)$ with $k$ in $\mathcal{K}$. In other words, there exist integers $y_{1}, \ldots, y_{4}$, not all zero, and an infinite subset $\mathcal{K}_{0}$ of $\mathcal{K}$ such that

$$
y_{1} b^{r_{k}+q_{k}}+y_{2} b^{r_{k}}+y_{3} m_{k}^{0} b^{r_{k}}+y_{4} m_{k}=0
$$

for every $k$ in $\mathcal{K}_{0}$. Dividing (11) by $b^{r_{k}}\left(b^{q_{k}}-1\right)$ and letting $k$ tend to infinity along $\mathcal{K}_{0}$, we get

$$
y_{1}+y_{3} \xi_{0}+y_{4} \xi_{1}=0
$$

This shows that $1, \xi_{0}$ and $\xi_{1}$ are linearly dependent over $\mathbb{Q}$, a contradiction with Theorem 3. Consequently, $\alpha_{0} \xi_{0}+\alpha_{1} \xi_{1}$ is transcendental. 


\section{Proof of Theorem 1}

Following $[5,10,11]$, we introduce the following function $\phi$.

Definition 2. Let $\lambda, \theta$ be real numbers in $(0,1)$. Let $\phi: \mathbb{R} \mapsto \mathbb{R}$ be the real function of the real variable $y$ defined by the formula

$$
\phi(y)=\phi_{\lambda, \theta}(y)=\frac{\delta(\lambda, \theta)}{1-\lambda}+(1-\lambda) \sum_{k \geq 0}\lfloor y-(k+1) \theta\rfloor \lambda^{k},
$$

where the value $\delta(\lambda, \theta)$ is given by $(1)$.

A detailled study of the function $\phi$ may be found in [11]. It satisfies the functional equation

$$
\{\phi(y+\theta)\}=f(\{\phi(y)\}), \quad \forall y \in \mathbb{R} .
$$

We deal here with the special case $\mu=1$ in the setting of [11].

The function $\phi$ enables us to parametrize the points of our Cantor set $C_{\lambda, \delta(\lambda, \theta)}$ introduced in Section 1. In the sequel, we assume that $\theta$ is an irrational number. Observe that $\phi$ is an increasing right continuous function which has a left discontinuity only at the points $l \theta+\mathbb{Z}$ for any positive integer $l$. We denote by $\phi\left(\{l \theta\}^{-}\right)$the left limit of $\phi$ at the point $\{l \theta\}$ for $l \geq 1$.

Lemma 4.1. For any real number $\lambda$ and any irrational $\theta$ with $0<\lambda, \theta<1$, we have the equality of sets

$$
C_{\lambda, \delta(\lambda, \theta)}=\overline{\phi(I)}=\phi([0,1]) \cup \bigcup_{l \geq 1} \phi\left(\{l \theta\}^{-}\right) .
$$

Moreover, the explicit formulae

$$
\begin{aligned}
\phi(\{l \theta\}) & =\frac{\left(1-\lambda^{l}\right) \delta(\lambda, \theta)}{1-\lambda}-\sum_{k=1}^{l} \lambda^{l-k}(\lfloor k \theta\rfloor-\lfloor(k-1) \theta\rfloor), \\
\phi\left(\{l \theta\}^{-}\right) & =\phi(\{l \theta\})-\lambda^{l-1}(1-\lambda), \\
\phi(\{-l \theta\}) & =\frac{1-\lambda^{-l}}{1-\lambda} \delta(\lambda, \theta)+\lambda^{-l}+\sum_{k=1}^{l-1} \lambda^{k-l}(\lfloor(k+1) \theta\rfloor-\lfloor k \theta\rfloor),
\end{aligned}
$$

hold for any integer $l \geq 1$.

Proof. It is established in Proposition 5 of [11] that

$$
\bigcap_{n \geq 1} f^{n}(I)=\phi(I)=I \backslash \bigcup_{l \geq 1}\left[\phi\left(\{l \theta\}^{-}\right), \phi(\{l \theta\})\right) .
$$

We deduce that

$$
C_{\lambda, \delta(\lambda, \theta)}=\overline{\phi(I)}=[0,1] \backslash \bigcup_{l \geq 1}\left(\phi\left(\{l \theta\}^{-}\right), \phi(\{l \theta\})\right)=\phi([0,1]) \cup \bigcup_{l \geq 1} \phi\left(\{l \theta\}^{-}\right),
$$

since $\phi$ is an increasing function with left discontinuities in $I$ at the points $\{l \theta\}, l \geq 1$. The formulae for $\phi(\{l \theta\})$ and $\phi\left(\{l \theta\}^{-}\right)$arise from Proposition 5 of [11]. For $\phi(\{-l \theta\})$, 
write

$$
\begin{aligned}
\phi(\{-l \theta\}) & =\frac{\delta(\lambda, \theta)}{1-\lambda}+(1-\lambda) \sum_{k \geq 0}\lfloor\{-l \theta\}-(k+1) \theta\rfloor \lambda^{k} \\
= & \frac{\delta(\lambda, \theta)}{1-\lambda}+\sum_{k \geq 0}(\lfloor\{-l \theta\}-(k+1) \theta\rfloor-\lfloor\{-l \theta\}-k \theta\rfloor) \lambda^{k} \\
= & \frac{\delta(\lambda, \theta)}{1-\lambda}+\sum_{k \geq 0}(\lfloor-(k+l+1) \theta\rfloor-\lfloor-(k+l) \theta\rfloor) \lambda^{k} \\
= & \frac{\delta(\lambda, \theta)}{1-\lambda}+\lambda^{-l} \sum_{k \geq l}(\lfloor-(k+1) \theta\rfloor-\lfloor-k \theta\rfloor) \lambda^{k} \\
= & \frac{\delta(\lambda, \theta)}{1-\lambda}-\lambda^{-l} \sum_{k \geq l}(\lfloor(k+1) \theta\rfloor-\lfloor k \theta\rfloor) \lambda^{k} \\
= & \frac{\delta(\lambda, \theta)}{1-\lambda}-\lambda^{-l} \sum_{k \geq 1}(\lfloor(k+1) \theta\rfloor-\lfloor k \theta\rfloor) \lambda^{k}+\lambda^{-l} \sum_{k=1}^{l-1}(\lfloor(k+1) \theta\rfloor-\lfloor k \theta\rfloor) \lambda^{k} \\
= & \frac{\delta(\lambda, \theta)}{1-\lambda}-\lambda^{-l}\left(\frac{\delta(\lambda, \theta)}{1-\lambda}-1\right)+\sum_{k=1}^{l-1}(\lfloor(k+1) \theta\rfloor-\lfloor k \theta\rfloor) \lambda^{k-l} \\
= & \frac{1-\lambda^{-l}}{1-\lambda} \delta(\lambda, \theta)+\lambda^{-l}+\sum_{k=1}^{l-1}(\lfloor(k+1) \theta\rfloor-\lfloor k \theta\rfloor) \lambda^{k-l} .
\end{aligned}
$$

This completes the proof of the lemma.

We are now able to apply Theorem 2. Lemma 4.1 tells us that an arbitrary element $z$ in $C_{\lambda, \delta(\lambda, \theta)}$ can be written either in the form $z=\phi(y)$ for some real number $y$ with $0 \leq y \leq 1$, or $z=\phi\left(\{l \theta\}^{-}\right)$for some integer $l \geq 1$. For any $y$ in $\mathbb{R}$, set

$$
\boldsymbol{x}_{y}=(\lceil y+(k+1) \theta\rceil-\lceil y+k \theta\rceil)_{k \geq 1} \in\{0,1\}^{\mathbb{N}} .
$$

The sequence $\boldsymbol{x}_{y}$ is Sturmian with slope $\theta$ and intercept $y+\theta$ modulo one. Moreover, $\boldsymbol{x}_{0}=\boldsymbol{c}_{\theta}$ is the characteristic Sturmian sequence of slope $\theta$ over $\{0,1\}$. Denote by

$$
\xi_{y, \lambda}=\sum_{k \geq 1}(\lceil y+(k+1) \theta\rceil-\lceil y+k \theta\rceil) \lambda^{k}
$$

the value at the point $\lambda$ of the power series whose sequence of coefficients is equal to $\boldsymbol{x}_{y}$. Then, we can write

$$
\delta(\lambda, \theta)=(1-\lambda)\left(1+\xi_{0, \lambda}\right),
$$

noting that $\lceil x\rceil=\lfloor x\rfloor+1$ for any $x$ in $\mathbb{R} \backslash \mathbb{Z}$, and

$$
\begin{aligned}
\phi(y)=\phi_{\lambda, \theta}(y) & =\frac{\delta(\lambda, \theta)}{1-\lambda}+(1-\lambda) \sum_{k \geq 0}\lfloor y-(k+1) \theta\rfloor \lambda^{k} . \\
& =\frac{\delta(\lambda, \theta)}{1-\lambda}+\lfloor y-\theta\rfloor+\sum_{k \geq 1}(\lfloor y-(k+1) \theta\rfloor-\lfloor y-k \theta\rfloor) \lambda^{k} \\
& =\frac{\delta(\lambda, \theta)}{1-\lambda}+\lfloor y-\theta\rfloor-\sum_{k \geq 1}(\lceil-y+(k+1) \theta\rceil-\lceil-y+k \theta\rceil) \lambda^{k} \\
& =1+\xi_{0, \lambda}+\lfloor y-\theta\rfloor-\xi_{-y, \lambda},
\end{aligned}
$$


since $\lfloor x\rfloor=-\lceil-x\rceil$ for any $x$ in $\mathbb{R}$. We immediately deduce from the above formulae that

$$
\phi(0)=0 \quad \text { and } \quad \phi(1)=1 .
$$

When $\lambda=1 / b$ for an integer $b \geq 2$ and $y$ is not congruent modulo one to some integer multiple $m \theta$ of $\theta$, the three numbers $1, \xi_{0, \lambda}, \xi_{-y, \lambda}$ are linearly independent over the field of algebraic numbers by Theorem 2 . Therefore, the non-zero linear combination

$$
\phi(y)=1+\xi_{0, \lambda}+\lfloor y-\theta\rfloor-\xi_{-y, \lambda}
$$

is a transcendental number. It remains to deal with points $y$ in $(0,1) \cap(\mathbb{Z} \theta+\mathbb{Z})$. These points have the form $y=\{m \theta\}$, for some non-zero integer $m$. Observe that, by a result of [10] quoted in Section 1, the real number $\delta(1 / b, \theta)$ is transcendental for any integer $b \geq 2$ and any irrational number $\theta$. Using the explicit formulae from the lemma, write

$$
\phi(\{m \theta\})=\frac{1-b^{-m}}{1-b^{-1}} \delta(1 / b, \theta)+A_{m},
$$

where

$$
A_{m}=\left\{\begin{array}{l}
-\sum_{k=1}^{m} b^{k-m}(\lfloor k \theta\rfloor-\lfloor(k-1) \theta\rfloor), \quad \text { if } \quad m \quad \text { is positive } \\
b^{-m}+\sum_{k=1}^{-m-1} b^{-k-m}(\lfloor(k+1) \theta\rfloor-\lfloor k \theta\rfloor), \quad \text { if } m \quad \text { is negative }
\end{array}\right.
$$

is a rational number. It follows that $\phi(\{m \theta\})$ is a transcendental number, since the coefficient $\left(1-b^{-m}\right) /\left(1-b^{-1}\right)$ is a non-zero rational number. Finally, $\phi\left(\{l \theta\}^{-}\right)=\phi(\{l \theta\})-$ $\lambda^{l-1}(1-\lambda)$ is also transcendental. Theorem 1 is proved.

\section{REFERENCES}

[1] V. Berthé, C. Holton, and L. Q. Zamboni, Initial powers of Sturmian sequences, Acta Arith. 122 (2006), 315-347.

[2] Y. Bugeaud, Dynamique de certaines applications contractantes, linéaires par morceaux, sur [0,1[, C. R. Acad. Sci. Paris Série I 317 (1993), 575-578.

[3] Y. Bugeaud and J.-P. Conze, Calcul de la dynamique d'une classe de transformations linéaires contractantes mod 1 et arbre de Farey, Acta Arith. 88 (1999), 201-218.

[4] Y. Bugeaud and D. H. Kim, A new complexity function, repetitions in Sturmian words, and irrationality exponents of Sturmian numbers, Trans. Amer. Math. Soc. 371 (2019), no. 5, 32813308.

[5] R. Coutinho, Dinâmica simbólica linear. Ph. D. Thesis, Instituto Superior Técnico, Universidade Técnica de Lisboa, February 1999.

[6] S. Ferenczi and Ch. Mauduit, Transcendence of numbers with a low complexity expansion, J. Number Theory 67 (1997), 146-161.

[7] N. P. Fogg, Substitutions in dynamics, arithmetics and combinatorics. Edited by V. Berthé, S. Ferenczi, C. Mauduit and A. Siegel. Lecture Notes in Mathematics, 1794. Springer-Verlag, Berlin, 2002.

[8] M. Hata, Neurons. A mathematical ignition. Series on Number Theory and its Applications, Vol. 9 (2015), World Scientific Publishing.

[9] S. Janson and C. Öberg, A piecewise contractive dynamical system and election methods. Preprint. arXiv:1709.06398v1 [Math. DS].

[10] M. Laurent and A. Nogueira, Rotation number of contracted rotations, J. Modern Dynamics 12 (2018), 175-191.

[11] M. Laurent and A. Nogueira, Dynamics of 2-interval piecewise affine maps and Hecke-Mahler series. Preprint. arXiv:1907.08655v1 [Math. DS].

[12] M. Lothaire, Algebraic combinatorics on words. Encyclopedia of Mathematics and its Applications, 90. Cambridge University Press, Cambridge, 2002.

[13] K. Mahler, Some suggestions for further research, Bull. Austral. Math. Soc. 29 (1984), 101-108.

[14] W. M. Schmidt, Norm form equations, Ann. of Math. 96 (1972), 526-551.

[15] W. M. Schmidt, Diophantine approximation. Lecture Notes in Mathematics 785, Springer, Berlin, 1980. 
YANN BUGEAUD (yann.bugeaud@math.unistra.fr) : Université de Strasbourg, IRMA, CNRS, UMR 7501, 7 rue René Descartes, 67084, Strasbourg, France.

DONG HAN KIM (kim2010@dongguk.edu) : Department of Mathematics Education, Dongguk University - Seoul, 30 Pildong-ro 1-gil, Jung-gu, Seoul, 04620 Korea.

MICHEL LAURENT (michel-julien.laurent@univ-amu.fr) : Aix-Marseille Université, CNRS, Centrale Marseille, Institut de Mathématiques de Marseille, 163 avenue de Luminy, Case 907, 13288, Marseille Cédex 9, France.

ARNALDO NOGUEIRA (arnaldo.nogueira@univ-amu.fr) : Aix-Marseille Université, CNRS, Centrale Marseille, Institut de Mathématiques de Marseille, 163 avenue de Luminy, Case 907, 13288, Marseille Cédex 9, France. 